\title{
Mandatory Resection of Strangulation Marks in Small Bowel Obstruction?
}

\author{
Samuel Andreas Käser • Niels Willi • \\ Christoph Andreas Maurer
}

Published online: 17 October 2013

(C) Société Internationale de Chirurgie 2013

\begin{abstract}
Background No evidence is available on how to treat intraoperatively detected band-shaped strangulation marks of the bowel wall originating from an adhesive band or hernia ring. The authors prefer to resect these hazardous strangulation marks to avoid secondary small bowel perforation. This retrospective study investigated the prevalence of intraoperatively unrecognized ulceration and transmural necrosis at the site of the strangulation marks. Methods From July 2003 to July 2011, a total of 31 of 461 patients with acute bowel obstruction underwent small bowel resection due to strangulation marks, exclusively. Seven patients had two strangulation marks, resulting in 38 strangulation marks to be analyzed.

Results From 38 examined strangulation marks, 14 (36.8\%) exhibited deep ulceration or transmural necrosis. Four $(10.5 \%)$ necrotic lesions had already been recognized intraoperatively, while 7 (18.4\%) unsuspicious strangulation marks showed deep ulceration and $3(7.9 \%)$ showed transmural necrosis exclusively at final histopathologic examination. The number of strangulation marks that needed to be resected for prevention of one missed deep ulceration
\end{abstract}

The results of this study were presented at the annual meeting of the Swiss Surgical Society, Davos, Switzerland, June 20-22, 2012.

S. A. Käser $(\bowtie) \cdot$ C. A. Maurer

Department of General, Visceral, Vascular, and Thoracic

Surgery, Hospital of Liestal, Affiliated to the University of

Basel, Liestal, Switzerland

e-mail: sakaeser@swissonline.ch

C. A. Maurer

e-mail: christoph.maurer@ksli.ch

N. Willi

Institute of Pathology, Liestal, Switzerland and/or transmural necrosis of the small bowel was 3.4. The presence of deep ulceration or transmural necrosis is associated with an obvious decrease in bowel diameter caudad to the strangulation mark. No anastomotic leak occurred.

Conclusion The severity of small bowel damage at the site of band-shaped strangulation marks may be underestimated by surgeons. The present series showed favorable results with a resection-per-principle policy for these strangulation marks. If an obvious decrease of bowel diameter aborally to the strangulation mark is present, resection or seromuscular invagination of the later is particularly recommended.

\section{Introduction}

The majority of cases of small bowel obstruction in Western countries are caused by postoperative adhesions while the rate of incarcerated hernias has declined [1-4]. Small bowel obstruction has a morbidity rate of up to $23 \%[1,2]$ and a mortality rate of up to $11 \%[1,3]$; thus, optimal therapy remains a challenge. One problem in small bowel obstruction is deciding whether bowel resection is required. However, the accuracy of clinical assessment of bowel viability is poor [5, 6]. Bowel resections result in longer operating time and higher rates of superficial wound infections [3], but missed intestinal wall injuries have a significant impact on mortality [1].

Although strangulation marks from an adhesive band or a hernia ring have been described in the literature [7-9], no randomized controlled trials on strangulation marks and no guidelines on how to handle them are currently available. This clinically important issue seems to be unexplored.

The treatment policy at the authors' department is to resect all strangulation marks in small bowel obstruction. 
This policy is based on the possibility of unrecognized ulceration and necrosis of the bowel wall due to local compression ischemia, which has the risk of secondary perforation or later fibrous stenosis if kept in situ.

The present study aimed to investigate the small bowel specimens with regard to the prevalence of intraoperatively unrecognized ulceration and necrosis at the site of the strangulation mark as surrogate markers for potential secondary bowel perforation.

\section{Methods}

Each surgeon at our institution has been instructed to resect all strangulation marks in small bowel obstruction. These strangulation marks are defined as a persistent, palpable, band-shaped impression with thinning and discoloration of the bowel wall from an adhesive band or a hernia ring. The presence of a strangulation mark is evaluated intraoperatively by palpation and inspection. The appearance of a typical strangulation mark is shown in Fig. 1. It is up to the surgeon to decide on the length of bowel to be resected. In the case of two strangulation marks, or in the case of the presence of serosal lesions after adhesiolysis, the length of bowel that needs resection is longer than that in the case of a single strangulation mark without further damage of the bowel wall. If there is no risk of short bowel syndrome, we prefer to resect a longer bowel segment which needs only one anastomosis instead of resecting two short segments and doing two anastomoses. As a rough guideline, we prefer to do only one anastomosis if no more than $20 \mathrm{~cm}$ of viable bowel has to be sacrificed to avoid two anastomoses and if the terminal ileum is not affected.

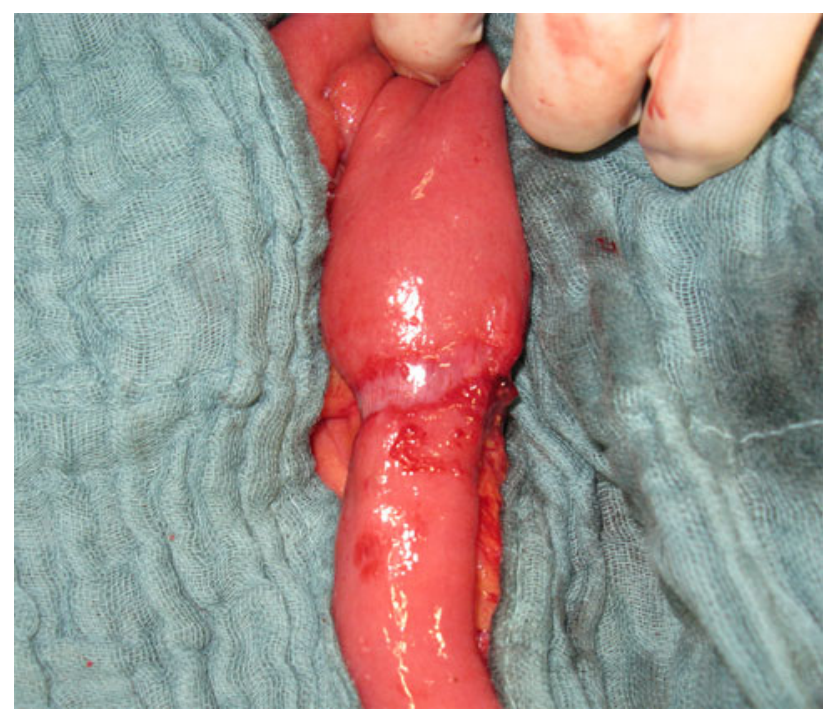

Fig. 1 A segment of small bowel with a typical strangulation mark from a fibrous band in small bowel obstruction
By doing an electronic search on all surgery reports at our institution from July 2003 to July 2011 using the search string "*ileu*," 461 consecutive patients who underwent surgery for bowel obstruction were identified. Exclusion criteria were large bowel obstruction $(n=81)$; small bowel obstruction without bowel resection needed $(n=182)$; small bowel resection for obstruction due to peritoneal carcinomatosis, small bowel cancer, anastomotic stenosis, or obstructive ileitis terminalis $(n=98)$, for strangulation of mesentery with consecutive infarction $(n=47)$, for unfeasible adhesiolysis $(n=20)$, and for Richter's hernia $(n=2)$. Thirty-one patients who had bowel resection just for the presence of strangulation marks (as explicitly mentioned in the operation report) remained. The surgeon usually estimated the length of the native nonstretched specimen. All surgical specimens were sent to pathology for examination. The pathologist measured the length of the specimens after fixation in formalin. There were seven patients who had two strangulation marks. Thus, 38 strangulation marks were resected and analyzed by the pathologist. Through chart review, the patients' characteristics and previous operations were retrospectively assessed. To verify the pathology report, each specimen was retrospectively judged again by a pathologist (N. Willi) and, if necessary, the pathology report was revised.

\section{Statistics}

Results are expressed as median and range. The relationships between predisposing factors and the presence of ulceration/transmural necrosis were analyzed with the Wilcoxon rank-sum test or Fisher's exact test. The Wilcoxon rank-sum test was used to analyze numerical data. A two-sided Fisher's exact test was used for categorical data. $p$ values $<0.05$ were considered significant.

\section{Results}

The patients' characteristics and previous operations are given in Table 1. There was no statistically significant relationship between ulceration/transmural necrosis and age $(p=0.633)$, gender $(p=0.277)$, previous operations $(p=0.401)$, cardiovascular comorbidities $(p=0.645)$, pulmonary comorbidities $(p=0.264)$, renal comorbidities $(p=0.330)$, cerebral comorbidities $(p=1.000)$, endocrine comorbidities $(p=0.598)$, or history of malignancy $(p=0.272)$.

The patients' symptoms and intraoperative findings are given in Table 2. There was no statistically significant relationship between ulceration/transmural necrosis and the presence of pain $(p=0.395)$ or emesis $(p=1.000)$, 
duration of symptoms $(p=0.0866)$, location $(p=0.658)$, previous operations $(p=0.401)$, the presence of incarcerated hernia $(p=0.440)$, and the presence of ascites $(p=0.722)$. However, the presence of an obvious decrease of bowel diameter caudad to the strangulation mark was significantly associated with the presence of ulceration/ transmural necrosis $(p=0.039)$.

In the case of a single strangulation mark as the reason for bowel resection $(n=19)$, the length of the native nonstretched resected bowel segments was $4-11 \mathrm{~cm}$ (median $=7 \mathrm{~cm})$. The length of these bowel segments after shrinkage in formalin was $3-9 \mathrm{~cm}$ (median $=5.5 \mathrm{~cm}$ ). In five other cases with additional reasons for bowel resection, such as serosal lesions due to adhesiolysis, the resected bowel segments were $14,30,35,35$, and $60 \mathrm{~cm}$ long. In the cases with two strangulation marks $(n=7)$, the length of the resected bowel segments was $5.5-38 \mathrm{~cm}$ (median $=12 \mathrm{~cm}$ ). The length of these bowel segments after shrinkage in formalin was $4-28 \mathrm{~cm}$ $($ median $=11 \mathrm{~cm})$.

Table 1 Patients' characteristics $(n=31)$

\begin{tabular}{ll}
\hline Male gender & $39 \%(12 / 31)$ \\
Age [median (range)] (years) & $76(18-93)$ \\
Previous abdominal operations & $74 \%(23 / 31)$ \\
Previous appendectomy & $23 \%(7 / 31)$ \\
Previous colon or rectal resection & $38 \%(12 / 31)$ \\
Previous gynecological operation & $23 \%(7 / 31)$ \\
Previous cholecystectomy/fundoplication & $10 \%(3 / 31)$ \\
Cardiovascular comorbidities & $58 \%(18 / 31)$ \\
Pulmonary comorbidities & $39 \%(12 / 31)$ \\
Renal insufficiency & $16 \%(5 / 31)$ \\
Cerebral comorbidities & $16 \%(5 / 31)$ \\
Endocrine comorbidities & $13 \%(4 / 31)$ \\
History of malignancy & $32 \%(10 / 31)$ \\
\hline
\end{tabular}

Table 2 Prevalence of symptoms and intraoperative findings

\begin{tabular}{lc}
\hline $\begin{array}{l}\text { Duration of symptoms [median } \\
\text { (range)] }\end{array}$ & 2 days (12 h to 14 days) \\
Prevalence of pain & $97 \%$ (30 of 31 patients) \\
Prevalence of emesis & $67 \%$ (21 of 31 patients) \\
Obvious decrease in bowel & $81 \%$ (25 of 31 patients) \\
$\quad$ diameter & $29 \%$ ( 9 of 31 patients) \\
Incarceration of hernia & $16 \%$ (5 of 31 patients) \\
Ascites & $18 \%$ ( 7 of 38 strangulation \\
Jejunum affected & marks) \\
& $82 \%(31$ of 38 strangulation \\
Ileum affected & marks) \\
Uncertain bowel viability & $34 \%$ (13 of 38 strangulation \\
& marks) \\
\hline
\end{tabular}

The number and prevalence of lesions found in the bowel segments with the resected strangulation marks $(n=38)$ are given in Table 3. Excluding the cases with obvious local bowel wall necrosis $(n=4)$, the number of strangulation marks that need to be resected for prevention of one missed transmural bowel necrosis was 11.4 (3/34), and for prevention of one missed transmural necrosis and/ or deep ulceration, the number was 3.4 (10/34). In the case of the presence of an obvious decrease of bowel diameter aborally to the strangulation mark, the risk of ulceration and/or transmural necrosis was $52 \%(13 / 25)$. The typical intraoperative and microscopic findings are shown in Figs. 1, 2, 3 .

The following postoperative complications were recorded: pneumonia $(n=8)$, urinary tract infection $(n=5)$, central venous catheter-related infection $(n=2)$, wound infection $(n=4)$, and death after postoperative bleeding in

Table 3 Number and prevalence of lesions found in the bowel segments with the resected strangulation marks

\begin{tabular}{lll}
\hline Type of lesion & $\begin{array}{l}\text { No. of strangulation marks } \\
\text { resected (total } n=38)\end{array}$ & $\begin{array}{l}\text { Prevalence } \\
(\%)\end{array}$ \\
\hline $\begin{array}{l}\text { Intraoperatively } \\
\text { apparent transmural } \\
\text { necrosis }\end{array}$ & $n=4$ & 11 \\
$\begin{array}{l}\text { Clinically unapparent } \\
\text { transmural necrosis }\end{array}$ & $n=3$ & 8 \\
$\begin{array}{l}\text { Deep ulceration } \\
\text { No severe lesion }\end{array}$ & $n=7$ & 18 \\
\hline
\end{tabular}

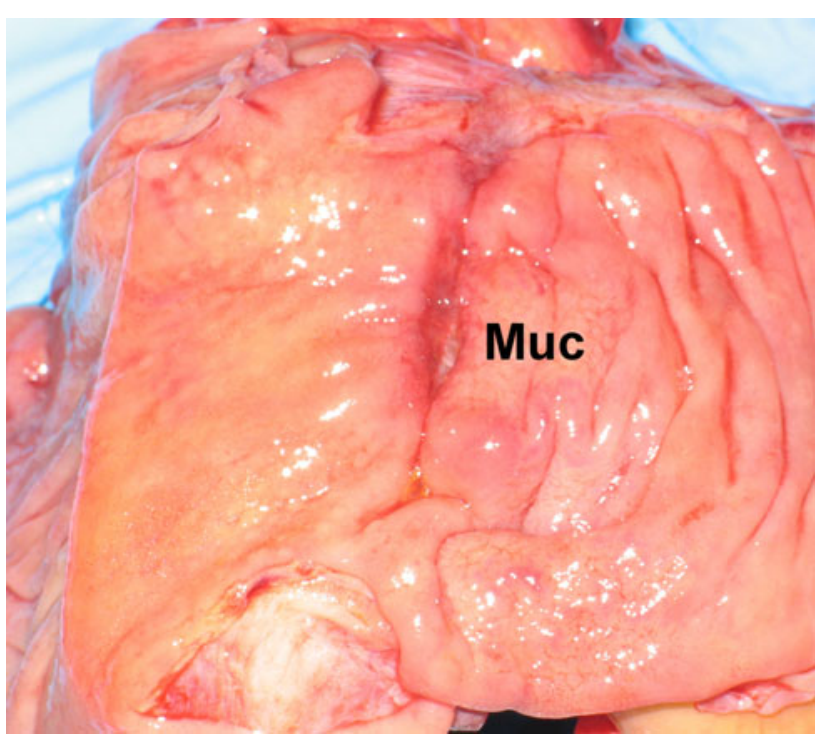

Fig. 2 The mucosa of an opened segment of small bowel with a strangulation mark from a fibrous band in small bowel obstruction. The typical brownish ulceration of the mucosa (Muc) can be seen at the site of compression 


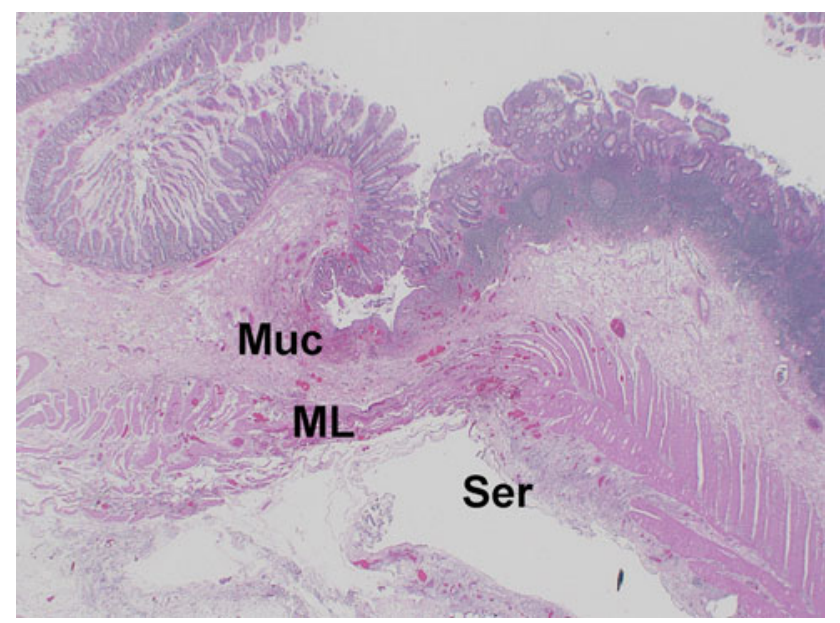

Fig. 3 A longitudinal section with hematoxylin-eosin staining of a small bowel segment with a strangulation mark from a fibrous band in small bowel obstruction. Ulceration of the mucosa (Muc) and submucosa, thinning of the muscle layer (ML) at the site of compression, and fibrosis of the serosa (Ser) in the neighborhood of the site of compression are exhibited (magnification $\times 12.5$ )

a patient who had taken oral anticoagulants $(n=1)$. There were no anastomotic leaks. Thus, the nonsurgical complication rate was $52 \%$, the surgical complication rate was $13 \%$, and mortality was $3 \%$ in the present series.

\section{Discussion}

In this study the prevalence of deep ulceration/transmural necrosis was determined in bowel segments with strangulation marks originating from an adhesive band or hernia ring in small bowel obstruction without strangulation of the mesentery. The significance of risk factors for deep ulceration/transmural necrosis was analyzed.

An unexpectedly high percentage $(36.8 \%)$ of the analyzed strangulation marks exhibited deep ulceration/transmural necrosis. Less than one-third of the cases with deep ulceration/transmural necrosis were already recognized intraoperatively. The clinical assessment of bowel viability is difficult $[5,6]$, and it is obviously impossible to determine the viability of the inner layers of the small bowel wall without performing an enterotomy. This explains why the number of strangulation marks that need to be resected for prevention of one missed deep ulceration/transmural necrosis was as low as 3.4.

As an alternative to resection some surgeons recommend a second-look laparotomy in the case of questionable bowel viability [10]. However, patients requiring a secondlook laparotomy for bowel perforation often need a stoma of the small bowel which has a high complication rate [11], and they often become critically ill and need a long time to recover. Usually, the segment of small bowel resected to remove a strangulation mark is short; therefore, the risk of causing short bowel syndrome is low [12]. Furthermore, the leakage rate of small bowel anastomosis is known to be very low (range 0-8.3\%) [13]. This is why surgeons should have a low threshold of suspicion of unrecognized bowel ischemia and resect any questionable bowel.

In general, the risks of bowel resection have to be balanced against the risks of misjudgment of bowel viability with secondary bowel perforation. The longer operating time and the higher rate of superficial wound infections in the case of resection [3] have to be balanced against the risks of a nonresected intestinal wall injury as an independent risk factor for complications and higher mortality rate [1]. Ulceration/transmural necrosis of the bowel can lead to bacterial invasion and perforation, or if healing is possible, it can lead to later development of fibrotic stenosis $[7,14]$. Considering the very low risk of an anastomotic leak [13], this can be avoided by resection of bowel segments with persistent strangulation marks. This is confirmed by our own results, without any anastomotic leak occurring. As known from the literature, the majority of postoperative complications in this study were nonsurgical $[1,15]$.

A valuable alternative to resection might be invagination with some seromuscular sutures to avoid the risk of an anastomotic leak in cases with a strangulation mark without obvious necrosis (personal communication by Dr. Moshe Shein, with kind permission).

From the analyzed predisposing factors for deep ulceration/transmural necrosis, only the presence of an obvious decrease of bowel diameter caudad to the compression site was significantly associated with deep ulceration/transmural necrosis. According to the law of Laplace, the dilatation of the bowel segment oral to the obstruction is proportional to the intraluminal pressure and proportional to the wall tension. We speculate that the high intraluminal pressure pressing the soft bowel wall against the firm obstructive band or hernia ring results in local ischemia. Bowel ischemia usually is pronounced in the intestinal mucosa [16-22]. This is why unsuspicious strangulation marks often show ulceration if the bowel is cut open. In the subgroup of patients with an obvious decrease of bowel diameter at the site of the strangulation mark, the risk of deep ulceration and/or transmural necrosis was more than $50 \%$. Therefore, resection of strangulation marks is highly recommended in patients with an obvious decrease of bowel diameter at the site of the strangulation mark.

There was only a tendency that duration of symptoms was associated with deep ulceration and/or transmural necrosis. The duration of bowel occlusion is known to be associated with deep ulceration and/or transmural necrosis [8]. If the duration of ischemia is short, then recovery of the normal structure of the bowel occurs within 24-72 h [17, 
22-24]. However, the mucosa is not capable of recovery after total ischemia of more than $7 \mathrm{~h} \mathrm{[25].}$

The ileum was affected in most cases $(82 \%)$. We speculate that this might be because previous abdominal surgery had been performed more often in the lower abdomen than in the mid or upper abdomen. Also, due to the longer and more mobile mesentery, the ileum is probably more prone to be captured by an adhesive band than the jejunum.

Because of the retrospective design of this study, it has several shortcomings. The main shortcoming is that it is missing a control group so the natural course of strangulation marks is not known. To address this, a randomized study is required. However, because of important ethical concerns for such a study, it is very unlikely that this randomized study will ever be conducted.

It cannot be excluded that some cases with strangulation marks have been missed in this retrospective setting. However, the predominance of women in the small bowel cases caused by adhesions and the prevalence of comorbidities are comparable to what is known from the literature [1]. Furthermore, the most common previous abdominal operations responsible for small bowel obstruction caused by adhesions were comparable to what is known from the literature (appendectomy $23 \%$, colorectal resection $21 \%$, gynaecological surgery $12 \%$, upper gastrointestinal surgery $9 \%$, and small bowel surgery $8 \%$ ) [2, 4]. Thus the population of this study seems to be representative.

\section{Conclusion}

The pathological examination of the resected small bowel segments bearing strangulation marks revealed deep ulcers or even transmural necrosis in an unexpected high percentage of specimens. If an obvious decrease of the bowel diameter aborally to the strangulation mark is present, resection or seromuscular invagination of a strangulation mark is highly recommended to reduce the risk of secondary perforation or fibrous stenosis.

\section{References}

1. Duron JJ, du Montcel ST, Berger A et al (2008) Prevalence and risk factors of mortality and morbidity after operation for adhesive postoperative small bowel obstruction. Am J Surg 195(6): 726-734

2. Fevang BT, Fevang J, Stangeland L et al (2000) Complications and death after surgical treatment of small bowel obstruction: a 35-year institutional experience. Ann Surg 231(4):529-537

3. Margenthaler JA, Longo WE, Virgo KS et al (2006) Risk factors for adverse outcomes following surgery for small bowel obstruction. Ann Surg 243(4):456-464
4. Cox MR, Gunn IF, Eastman MC et al (1993) The operative aetiology and types of adhesions causing small bowel obstruction. Aust N Z J Surg 63(11):848-852

5. Karliczek A, Harlaar NJ, Zeebregts CJ et al (2009) Surgeons lack predictive accuracy for anastomotic leakage in gastrointestinal surgery. Int J Colorectal Dis 24:569-576

6. Horgan PG, Gorey TF (1992) Operative assessment of intestinal viability. Surg Clin North Am 72(1):143-155

7. Hofmann M (1907) Das Verhalten des Darmes bei Incarceration, insbesondere an den Schnürfurchen. Beitr Klin Chir 54:85-160

8. Van Beuren FT (1920) The relation between intestinal damage and delayed operation in acute mechanical ileus. Ann Surg 72: 610-615

9. Lehr L, Siewert JR (1992) Ileus. In: Kremer K, Lierse W, Platzer W, Schreiber HW, Weller S (eds) Chirurgische Operationslehre, vol 6, 1st edn. Thieme, Stuttgart, p 332

10. Meng X, Liu L, Jiang $H$ (2010) Indications and procedures for second-look surgery in acute mesenteric ischemia. Surg Today 40:700-705

11. Robertson I, Leung E, Hughes D et al (2005) Prospective analysis of stoma-related complications. Colorectal Dis 7(3):279-285

12. Wilmore DW, Robinson MK (2000) Short bowel syndrome. World J Surg 24(12):1486-1492. doi:10.1007/s002680010266

13. Chow A, Tilney HS, Paraskeva P et al (2009) The morbidity surrounding reversal of defunctioning ileostomies: a systematic review of 48 studies including 6,107 cases. Int J Colorectal Dis 24:711-723

14. Bougard V, Avisse C, Patey M et al (2008) Double ischemic ileal stenosis secondary to mesenteric injury after blunt abdominal trauma. World J Gastroenterol 14:143-145

15. Dindo D, Demartines N, Clavien PA (2004) Classification of surgical complications: a new proposal with evaluation in a cohort of 6336 patients and results of a survey. Ann Surg 240(2): 205-213

16. Ruf W, Suehiro GT, Suehiro A, Pressler V, McNamara JJ (1980) Intestinal blood flow at various intraluminal pressures in the piglet with closed abdomen. Ann Surg 191(2):157-163

17. Robinson JW, Mirkovitch V, Winistörfer B, Saegesser F (1981) Response of the intestinal mucosa to ischaemia. Gut 22(6): 512-527

18. Brown RA, Chiu CJ, Scott HJ, Gurd FN (1970) Ultrastructural changes in the canine ileal mucosal cell after mesenteric arterial occlusion. Arch Surg 101(2):290-297

19. Vákonyi T, Wittmann T, Varró V (1977) Effect of local circulatory arrest on the structure of the enterocytes of the isolated intestinal loop. Digestion 15(4):295-302

20. Chiu CJ, McArdle AH, Brown R, Scott HJ, Gurd FN (1970) Intestinal mucosal lesion in low-flow states. I. A morphological, hemodynamic, and metabolic reappraisal. Arch Surg 101(4): 478-483

21. Menge H, Robinson JW (1979) Early phase of jejunal regeneration after short term ischemia in the rat. Lab Invest 40(1):25-30

22. Wagner R, Gabbert H, Höhn P (1979) The mechanism of epithelial shedding after ischemic damage to the small intestinal mucosa. A light and electron microscopic investigation. Virchows Arch B 30(1):25-31

23. Robinson JW, Mirkovitch V (1972) The recovery of function and microcirculation in small intestinal loops following ischaemia. Gut 13(10):784-789

24. Cameron GR, Khanna SD (1959) Regeneration of the intestinal villi after extensive mucosal infarction. J Pathol Bacteriol 77(2): 505-510

25. Pitha J (1971) The fine structure of regenerating epithelium in the small intestine. Virchows Arch B 7(4):314-341 